\title{
Atenção Básica em Saúde Bucal: a experiência no curso de graduação
}

Primary Dental Care: the experience in the undergraduate courses

Lara Jansiski Motta ${ }^{1}$, Patrícia Elaine Gonçalves ${ }^{1}$, Marcia Cristina Lopes ${ }^{1}$

'Universidade Nove de Julho (UNINOVE) - São Paulo (SP), Brasil.

DOI: http://dx.doi.org/10.7322/abcshs.v40i3.815

\section{RESUMO}

Introdução: A participação do cirurgião-dentista na atenção básica fomenta a importância da formação do profissional de clínica geral como generalista, bem como a necessidade do perfil de um profissional de saúde, com olhar holístico sobre o paciente. Relato de experiência: O presente artigo tem como objetivo relatar a experiência vivenciada na implantação da Clínica de Atenção Básica no curso de Odontologia (CAB). A análise reflexiva da experiência vivida se deu por meio do relato dos discentes participantes do projeto. Conclusão: A nova proposta de atendimento, uniu a teoria com as práticas técnicas tão importantes para o discente, permitindo que os discentes ampliassem os seus conhecimentos quando expostos à situações e processo de trabalho compatíveis com a realidade da comunidade e do serviço.

Palavras-chave: atenção básica à saúde; saúde pública; odontologia; ensino.

\section{ABSTRACT}

Introduction: The activities developed promote the importance of professional training general practitioners, generalist, as well as the need for access to a health professional with a comprehensive view. Experience report: This study aims to report an experience about implementation of the Primary Care at the dental clinic. The reflective analysis of this experience was through the story of students. Conclusion: This new proposal united theory with practical techniques as important to the student, allowing students broaden their knowledge when exposed to situations and work process compatible with the reality of community and service.

Keywords: primary health care; public health; dentistry: teaching. 


\section{INTRODUÇÃO}

A formação prática dos cirurgiões-dentistas está relacionada às atividades das disciplinas curriculares básicas em laboratórios experimentais. Esta forma, repetida ano após ano, não gerou a tão esperada articulação entre a metodologia clínica e o método experimental das disciplinas curriculares básicas. Frente a esse quadro, uma lacuna estrutural se abre entre os dois ciclos: o básico e o profissionalizante. O enfoque principal, então, é dado à doença, seguindo o modelo biomédico individualista e a formação de profissional restrito a conhecimentos e práticas fragmentadas, tendendo para especializações precoces e com baixo alcance populacional ${ }^{1}$.

Roncalli $^{2}$ discorre que, durante as últimas décadas, alguns estudos epidemiológicos de âmbito nacional foram desenvolvidos com a finalidade de identificar os problemas de saúde bucal que acometem a população brasileira e os principais fatores de risco associados para propor medidas que solucionem estes problemas. $O$ acesso a serviços foi observado como de baixo alcance populacional, o que não condiz com o grande número de cirurgiões-dentistas no Brasil. Observou-se que os profissionais que atuam no mercado estão concentrados em determinadas regiões, nas grandes metrópoles e atuando principalmente no mercado privado. Já aqueles que atuam no Sistema Único de Saúde (SUS) muitas vezes reproduzem a prática do consultório privado com foco no modelo biomédico, tecnicista e individualista. Durante anos, o ensino da Odontologia foi marcado por uma visão biológica e tecnicista, com a valorização exagerada dos procedimentos técnicos e das clínicas de ensino fragmentado, com grande tendência de especialização precoce sem se preocupar com as necessidades da população ${ }^{3}$.

A partir deste contexto, começou, então, a se configurar um novo paradigma de ações de saúde bucal no país. O profissional de saúde não deve ter o seu foco de atuação apenas no atendimento individual, com visão clínica restrita a odontotécnica, mas, sim, deve ser preparado para as necessidades das pessoas, das famílias e da comunidade e para a mudança do paradigma de atenção. As mudanças no perfil epidemiológico das doenças bucais, as novas práticas baseadas em evidencias cientificas e, principalmente, a promoção de saúde no seu conceito ampliado exigem a formação de um profissional generalista, tecnicamente competente e com sensibilidade social com foco nas famílias para trabalhar no Setor público que apresenta a Estratégia de Saúde da Família como reorientadora destes serviços ${ }^{3,4}$.

A formação de profissionais mais capazes de desenvolverem uma assistência humanizada e alta qualidade e resolutividade serão impactantes até mesmo para os custos do SUS. O Brasil tem uma notável experiência em aproximação entre a academia e serviços, mas essa ainda está muito aquém do que seria necessário ${ }^{5}$.

Segundo Loretto et al. ${ }^{6}$, o discente em Odontologia deve ser direcionado ao treinamento na formação e desenvolvimento de habilidades e competências clinicas em clínicas de atenção hierarquizadas denominadas Clínica de Atenção Básica (CAB), Clínica de Atenção de Média Complexidade (CAMC) e Clínica de Atenção de Alta Complexidade (CAAC). A ideologia dessas clínicas devem fazer parte do projeto político-pedagógico (PPP) do curso, superando a formação em clínicas estanque, desarticuladas da realidade social e que refletiam ainda o modelo flexnerianobiologicista.

É de competência das instituições de ensino desenvolver atividades práticas que complementem o aprendizado teórico e que apresentem a realidade dos serviços de saúde pública ao acadêmico de Odontologia, a fim de que o aluno esteja preparado para raciocinar criticamente sobre os problemas sociais de sua comunidade. Tal atitude é fomentada quando o discente vivencia ações de atenção básica, corroborando com a Política Nacional de Saúde Bucal7, que tem priorizado os serviços de atenção básica em Odontologia.

O Objetivo deste Artigo é relatar a experiência da implantação da Clínica de Atenção Básica (CAB) no curso de graduação de Odontologia da Universidade Nove de Julho (UNINOVE). Relatos como este provocam a reflexão para a busca da melhor maneira de preparar o aluno da graduação para atuação eficiente no serviço de saúde. Diferentes experiências vivenciadas pelas Instituições de Ensino, pelos docentes e pelos discentes, devem ser compartilhadas para que o aprimoramento do processo de ensino-aprendizagem seja contínuo, tendo como foco principal o melhor preparo do futuro profissional de saúde.

De acordo com o exposto, torna-se fundamental descrever a experiência dos discentes inseridos na $\mathrm{CAB}$ e refletir nos avanços e desafios do projeto no processo pedagógico em saúde.

\section{RELATO DE EXPERIÊNCIA}

Este artigo se deu a partir da reflexão sobre grade curricular do curso de Odontologia da UNINOVE, seguindo pela proposta de implantação da Clínica de Atenção Básica (CAB) e, após a implantação, a análise reflexiva da experiência dos discentes neste projeto de mudança de cenário pedagógico. O presente estudo foi submetido ao Comitê de Ética em Pesquisas e obteve parecer favorável (n 768001/2013) e todos os participantes assinaram previamente o Termo de Consentimento Livre e Esclarecido.

A Clínica de Atenção Básica (CAB) foi implantada em maio 2013 no curso de graduação de Odontologia da Universidade Nove de Julho (UNINOVE), como uma proposta desafiadora para todo o grupo docente, em especial aos das disciplinas de Odontologia Preventiva Comunitária e Políticas de Saúde. A inserção da mesma foi realizada em parceria com a Clínica Odontológica Integrada, na qual os alunos apresentam forte inclinação para procedimentos clínicos especializados. Contudo, a inserção da CAB fomenta a importância da formação do 
profissional de clínica geral, como generalista, bem como a necessidade do perfil de um profissional de saúde, com olhar holístico sobre o paciente.

O curso de Odontologia da UNINOVE iniciou suas atividades em fevereiro de 2002, oferecido como bacharelado em regime seriado semestral, com período de duração de quatro anos, em oito semestres. A matriz curricular do curso, além das características de Estágio Supervisionado, Trabalho de Conclusão do Curso, Atividades Complementares, desenvolve o conteúdo de atenção às Políticas Públicas com maior profundidade. No entanto, ao analisar a grade curricular, o núcleo docente estruturante (NDE) observou algumas dificuldades enfrentadas pelos alunos para a aplicação do conteúdo teórico na prática clínica nos estágios supervisionados nos ambulatórios da instituição, pois as atividades clínicas eram realizadas de acordo com a necessidade individual do paciente e conforme a especialidade desta necessidade. Os pacientes passavam por triagem inicial para alocação, conforme a especialidade que atenderia a sua necessidade. Esta prática resultava no primeiro contato paciente-graduando, após a avaliação inicial de outro profissional que já havia determinado quais as necessidades do paciente. Nesta configuração, o aluno não colocava em prática a visão generalista para tomar a decisão e realizar o diagnóstico, apreciando a visão centrada no diagnostico integral, na promoção de saúde, na prevenção e no cuidado com as pessoas, considerando as questões socioculturais e econômicas e refletindo no núcleo familiar em que vive o indivíduo.

Observou-se, portanto, a necessidade de reorganizar o cenário pedagógico nos espaços internos da instituição, proporcionando uma vivência diária do processo de trabalho integral e não somente nas atividades desenvolvidas nas práticas externas em parceria com o serviço público. Para esta reorganização, foi implantado o projeto da $\mathrm{CAB}$ como instrumento formativo dos discentes. A clínica foi implantada nos dois últimos períodos do curso em parceria com a disciplina de Clínica Integrada, pois, neste momento, o aluno já passou por todas as disciplinas específicas e está apto a realizar todos os procedimentos de atenção básica em odontologia.

As atividades da CAB envolvem as ações de cadastramento das famílias e acompanhamento destas famílias em todo o tratamento odontológico e encaminhamento e acompanhamento para outras especialidades nos Ambulatórios Integrados de Saúde (AIS) da instituição.

Para que o aluno conheça as características dos pacientes, realize o cadastramento das famílias e coloque em prática a identificação da necessidade de realização dos procedimentos odontológicos de atenção básica e necessidade de encaminhamento para especialidade, são aplicados formulários específicos: a Ficha A Família, Ficha Individual e Ficha de referência e contra-referência.

O formulário elaborado para a Ficha A coleta informações como identificação da família, cadastro de todos os seus membros, situação de moradia e outras informações adicionais, que permitem à equipe de saúde conhecer as condições de vida das pessoas da sua área de abrangência e melhor planejar suas intervenções ${ }^{8}$. Já a Ficha
Individual, que é aplicada a cada integrante da Família, apresenta perguntas específicas sobre utilização de serviços odontológicos e morbidade bucal referida, autopercepção, impactos em saúde bucal e avaliação da saúde bucal. Sobre os dados de exame de índices epidemiológicos de saúde bucal, os alunos que integram a $\mathrm{CAB}$ são submetidos a uma capacitação/calibração sobre os índices, tais como cárie, doença periodontal, trauma, fluorose e indicadores de uso e necessidade de prótese.

Diante do material coletado, o aluno pode analisar a relação do perfil socioeconômico da família com os valores dos índices epidemiológicos avaliados, com o intuito de reiterar qual perfil familiar requer mais atenção à saúde bucal, e, assim, promover equidade.

A ficha de referência e contra-referência trata-se do encaminhamento dos pacientes de acordo com procedimentos que não são executados na $\mathrm{CAB}$, ou seja, procedimentos de média complexidade. Há o preenchimento de ficha de referência para cada tratamento especializado e encaminhado à clínica de especialidades necessária. Após a execução/finalização do tratamento o paciente retorna para acompanhamento na $\mathrm{CAB}$, que corresponde a resolutividade das ações em saúde bucal.

Para analisarmos esta experiência, consideramos o processo ensino-aprendizagem vivenciado pelos discentes no cenário pedagógico proposto. Foram coletados relatos da experiência dos docentes envolvidos na $\mathrm{CAB}$ e dos alunos inseridos no projeto. A análise reflexiva dos depoimentos leva a uma necessidade constante de reorganização dos processos no ensino e formação dos profissionais de saúde. O projeto teve como uma das preocupações possibilitar a aplicação prática da teoria relacionada aos conceitos e atenção à saúde, seguindo os princípios do SUS nos ambulatórios da instituição. A vivência e conhecimento dos processos de diagnóstico integral, organização dos serviços e o processode trabalho no serviço público puderam ser observadas nas falas dos discentes:

[...] A CAB teve grande colaboração no meu aprendizado pois, através dela, pude colocar em prática a teoria do aprendizado teórico ministrado nos primeiros semestres em Odontologia Preventiva e Comunitária. $\mathrm{Na} \mathrm{CAB}$, pude ter noção do funcionamento de parte das políticas de saúde pública no Brasil. O diferencial foi perceber que podemos atuar não só como odontologia curativa mas também preventiva, buscando, através dos dados levantados das famílias, encontrar métodos de prevenção para os problemas bucais (aluno, $7^{\circ}$ período).

A CAB me ajudou a desenvolver uma visão de estudo, onde demonstrou os princípios da gestão e das leis que regulamentam o desenvolvimento de trabalho aplicado no SUS. O diferencial no meu aprendizado foi a demonstração prática do atendimento na atenção básica na clínica da universidade da mesma forma que é apresentada em postos de saúde/unidades básicas de saúde, seguindo padrões de cadastramento e classificação de cada individuo, de forma coletiva (familiar), ou mesmo individual, 
desenvolvendo em nosso atendimento, técnicas de avaliação e classificação das prioridades de atendimento (aluno, $7^{\circ}$ período).

[...] em relação à abordagem dos pacientes, o que mais aprendi foi focar, interpretar a causa, descobrir de onde vem o problema, que pode ser familiar como: dieta familiar, orientação, moradia, etc. (aluno, $7^{\circ}$ período).

[...] na CAB, aprendemos a Aplicar basicamente as estratégias de baixa complexidade, orientando e buscando respostas para os diagnósticos dentro de sua família e sua vida (aluno, $7^{\circ}$ período).

[...] devemos prestar atenção nos sinais que os pacientes podem apresentar. Muitas vezes, os pacientes não contam sobre os problemas de saúde que realmente têm e, na CAB, tive a oportunidade de vivenciar uma situação com uma paciente portadora de HIV (aluno, $8^{\circ}$ período).

aprendi o quanto esses pacientes valorizam o atendimento humanizado, e que é possível 'educar' uma família inteira quando conseguimos fazer com que um de seus membros compreenda as informações que passamos. Valorize essas informações, se proponha a adquirir ou, até mesmo, mudar hábitos e ainda transmitir e compartilhar essa sua experiência com os outros (aluno, $8^{\circ}$ período).

Respeito ao próximo, saber escutar o paciente, dar atenção necessária, entender que o paciente que se encontra ali está porque precisa e, com certeza, um atendimento mais humanizado fará com que ele se sinta muito confortável e contente em voltar às próximas consultas e indicar aos amigos e familiares foi o que mais aprendi na $\mathrm{CAB}$ (aluno, $7^{\circ}$ período).

\section{DISCUSSÃO}

A formação acadêmica vem sofrendo mudanças que enfatizam a necessidade não só da teoria, mas também da prática, e todas as nuances que compõem a atenção básica. Neste quesito, observamos que a $\mathrm{CAB}$ colaborou muito com a vivência do graduando frente a problemas diagnosticados nas famílias. A prática de conhecer e classificar o risco familiar e individual também resgata conceitos sobre o processo saúde doença, o que faz com que aconteça naturalmente uma reflexão sobre a importância do núcleo familiar para a tomada de decisões nos tratamentos.
A análise reflexiva da fala dos discentes demonstra, nesta experiência, que é possível, ao mudar o cenário pedagógico, esperar um profissional egresso com uma nova postura, com compromissos éticos com a sociedade, ajudando o usuário a viver com saúde e que possa atuar em equipes multidisciplinares ${ }^{9}$.

A visão integral do paciente também é uma característica importante do cirurgião dentista generalista. Observou-se com os depoimentos que a $\mathrm{CAB}$ auxiliou no desenvolvimento da habilidade de interagir e interpretar a complexa realidade do núcleo familiar. Esta competência do profissional é um desafio durante a formação acadêmica, pois o processo ensino-aprendizagem deve sair do modelo centrado no diagnóstico de doenças, tratamento e recuperação para o centrado no diagnóstico integral, promoção de saúde, na prevenção e no cuidado com as pessoas, conforme proposto por Morita e Kriger $^{10}$.

A CAB deixa claro aos discentes, através do atendimento às famílias, os princípios que regem o nosso SUS, como a hierarquização, equidade no atendimento e integralidade. No entanto, apesar de seguir este modelo, essa clínica não é entendida como apenas "serviço público", mas uma forma generalista de atendimento e que pode ser replicada para pequenos grupos atendidos na sua futura vida profissional, quer seja pública ou privada. Esta visão possibilita o entendimento da realidade local e o exercício para o planejamento das atividades para enfrentamento dos fatores que colocam as famílias em risco, evitando complicações e encaminhamentos desnecessários para especialistas ${ }^{11,12}$.

Segundo Loretto et al. ${ }^{6}$, ao elaborar seu PPP, uma instituição pesquisada apresentou seu modelo de formação no qual se inclui a Clínica de Atenção Básica como primeira experiência clínica do aprendiz, situada no $5^{\circ}$ período do curso, e concluiu que houve a melhoria na triagem. A interdisciplinaridade e o compromisso e competência docente são as melhorias mais exigidas pelos alunos; e o fortalecimento da interdisciplinaridade e a organização dos prontuários são as melhorias mais exigidas pelos docentes. Nesse sentido, avançamos quanto a uma nova proposta de atendimento que uniu a teoria com as práticas técnicas tão importantes para o discente.

Acreditamos que o desafio maior será a integração das ações extras odontológicas, como clínicas médicas, psicologia e todos os demais cursos que a instituição oferece. Desta forma, nossas perspectivas são de ganhos através dos prontuários integrados $\mathrm{e}$ familiares, onde poderemos, em breve, trabalhar plenamente com a promoção, prevenção e recuperação da saúde.

A nova proposta de atendimento uniu a teoria com as práticas técnicas, tão importantes para o discente, permitindo que os discentes ampliassem os seus conhecimentos quando expostos à situações e processo de trabalho compatíveis com a realidade da comunidade e do serviço. 


\section{REFERÊNCIAS}

1. Zanetti CHG. A formação do cirurgião-dentista. In: Dias $A A$, organizador. Saúde Bucal Coletiva: metodologia de trabalho e práticas. São Paulo: Editora Santos; 2006; p. 21-41.

2. Roncalli AG. Projeto SB Brasil 2010: Pesquisa Nacional de Saúde Bucal revela importante redução da carie dentaria no pais. Cad Saúde Pública. 2011;27(1):4-5. http://dx.doi.org/10.1590/S0102-311X2010000300001

3. Moyses ST, Moyses SJ, Kriger L, Schmitt EJ. Humanizando a educação em Odontologia. Rev Abeno. 2003;3(1):58-64.

4. Kriger L. Perfil profissional para o programa Saúde da Família. In: Carvalho ACP, Morita MC, Kriger L. Políticas Pùblicas para Educação e Saúde em Odontologia. São Paulo: Nupps/USP; 2007; p.11-8.

5. Cruvinel VRN, Franco EJ, Bezerra L, Alves MM, Miranda AF, Carvalho DR. A formação do cirurgião-dentista generalista na Universidade Católica de Brasília. Rev Abeno. 2010;10(2):12-9.

6. Loretto NRM, Silva IDPP, Batista LC. Clínica de atenção básica: do discurso teórico à realidade prática. Rev Abeno. 2010;10(2):20-26.
7. Brasil. Ministério Da Saúde. Secretaria de Atenção a Saúde. Departamento de Atenção Básica. Coordenação Nacional de Saúde Bucal. Diretrizes da Política Nacional de Saúde Bucal. Brasília: 2004; p.16

8. Brasil. Ministério da Saúde. Secretaria de Atenção à Saúde. Departamento de Atenção Básica. SIAB: Manual do sistema de Informação de Atenção Básica. Brasília: Ministério da Saúde; 2003.

9. Pinheiro FMC, Nobrega-Therrien SM, Almeida MEL, Almeida MI A formação do cirurgião-dentista e a promoção de saúde no PSF Rev Odontol UNESP. 2008;37(1): 69-77.

10. Morita MC, Kriger L. Mudanças nos cursos de Odontologia e a interação com o SUS. Rev Abeno. 2004;4(1):17-21.

11. Lucas SD, Palmier AC, Amaral JHL, Werneck MAF, Senna MIB. Inserção do aluno de odontologia no SUS: contribuição do Pro Saúde. Rev Abeno. 2011;11(1):29-34

12. Moura TP, Costa RCM, Damasceno TC, Nuto SAS, Pequeno LL. Percepção da inserção de alunos na atenção primária: visão dos gestores e profissionais da Estratégia Saúde da Família. Rev Abeno. 2010;10(2):46-52. 